\title{
Recombinant tissue plasminogen activator in two patients with basilar artery occlusion
}

\author{
D Herderscheê, M Limburg, A Hijdra, P A Koster
}

\begin{abstract}
Two patients with angiographically proved basilar artery occlusion were treated with systemic recombinant tissue plasminogen activator (rtPA) according to protocol. The first patient was in a locked-in state and gradually deteriorated. On repeat angiography the basilar artery remained occluded. He died and necropsy revealed a pontine haemorrhagic infarction. The second patient, who was comatose and with decerebrate posturing, made a remarkable recovery. Angiography showed reperfusion. Therapy was initiated in the first patient after six hours and in the second after two hours. Treatment with rtPA is promising but probably not feasible for every patient. Success may depend on duration of occlusion and composition of occluding thrombus.
\end{abstract}

In patients with a severe neurological deficit and an angiographically proved basilar artery occlusion, prognosis for survival with a reasonable quality of life is poor. ${ }^{12} \mathrm{~A}$ recent case series of patients with basilar artery occlusion strongly suggested a relationship between induced reperfusion and beneficial clinical outcome. ${ }^{3}$ A case history suggested clot lysis with local recombinant tissue plasminogen activator (rtPA) therapy, but angiography was only repeated after 20 hours when spontaneous reperfusion may have occurred. ${ }^{4}$ We studied the efficacy and safety of systemic thrombolytic therapy with rtPA in two patients with severe neurological deficit and basilar artery occlusion.

Academisch Medisch Centrum, Amsterdam, The Netherlands. Department of Neurology D Herderscheê M Limburg A Hijdra

Department of Diagnostic Radiology P A Koster

Correspondence to: $\mathrm{Dr}$ Herderscheê, Department of Neurology H22-214, Academisch Medisch Centrum, $1105 \mathrm{AZ}$ Amsterdam, The Netherlands.

Received 5 March 1990 Accepted 24 May 1990

Patients and methods

Patients with severe neurological deficit suggesting extensive brainstem ischaemia, that is, coma or tetraparesis combined with cranial nerve dysfunction or a locked-in state, were eligible. Inclusion criteria were: time between onset of symptoms and rtPA treatment not more than six hours, age between 18 and 75 years, computerised tomography (CT) scan of the posterior fossa without signs of haemorrhage, angiographically proved occlusion of the basilar artery, and informed consent by a first degree relative.

Angiography was performed with selective catheterisation of the vertebral arteries after introduction of the catheter in the femoral artery. A total dose of $100 \mathrm{mg}$ rtPA was administered intravenously: a bolus of $10 \mathrm{mg}$ was followed by $50 \mathrm{mg}$ in the first hour and 20 $\mathrm{mg}$ in the second.and third hours, as already shown to be effective in cardiological practice. ${ }^{5}$ Subsequently $500 \mathrm{mg}$ acetylsalicylicacid (ASA) was administered intravenously and anticoagulation was continued with heparin, to prevent reocclusion as rtPA is a short acting agent. ${ }^{6}$ Angiography was repeated six hours after the first examination. Repeat CT was performed after 24 hours, one week and otherwise when indicated.

\section{Case histories}

Patient 1 A 59 year old man suddenly became comatose. He had a history of diabetes mellitus; retinopathy, cardiac failure, atrial fibrillation, peripheral arterial disease and renal failure necessitating chronic ambulatory peritoneal dialysis (CAPD). Shivering extensor movements and incontinence of urine occurred at the onset of symptoms. An initial diagnosis of an epileptic seizure was rejected when a second physical examination demonstrated a locked-in syndrome with intact vertical eye movements, paralysis of pontine cranial nerves, and a tetraparalysis. Angiography showed basilar artery occlusion after the origin of the anterior inferior cerebellar artery with extensive collateral circulation along the posterior inferior cerebellar artery to the superior cerebellar artery. Treatment with rtPA was initiated at six hours after onset and was complicated by a minor nose bleed and bleeding from the puncture site of the angiography, which could be stopped by local pressure. Neurological status remained unchanged and so was the repeat angiography.

A gradual deterioration set in and the patient died 26 hours after onset of symptoms. At necropsy there was no internal bleeding. The middle third of the basilar artery, showing irregular intimal thickening, was occluded by a thrombus, already showing signs of organisation. There was extensive haemorrhagic infarction of the pons and a haematoma with extension into the fourth ventricle.

Patient 2 A 36 year old man suddenly became dizzy while working, and was brought straight to the emergency ward. He had a worsening brainstem syndrome with dysarthria, eye movement abnormalities and a left hemiparesis, evolving into a coma with 

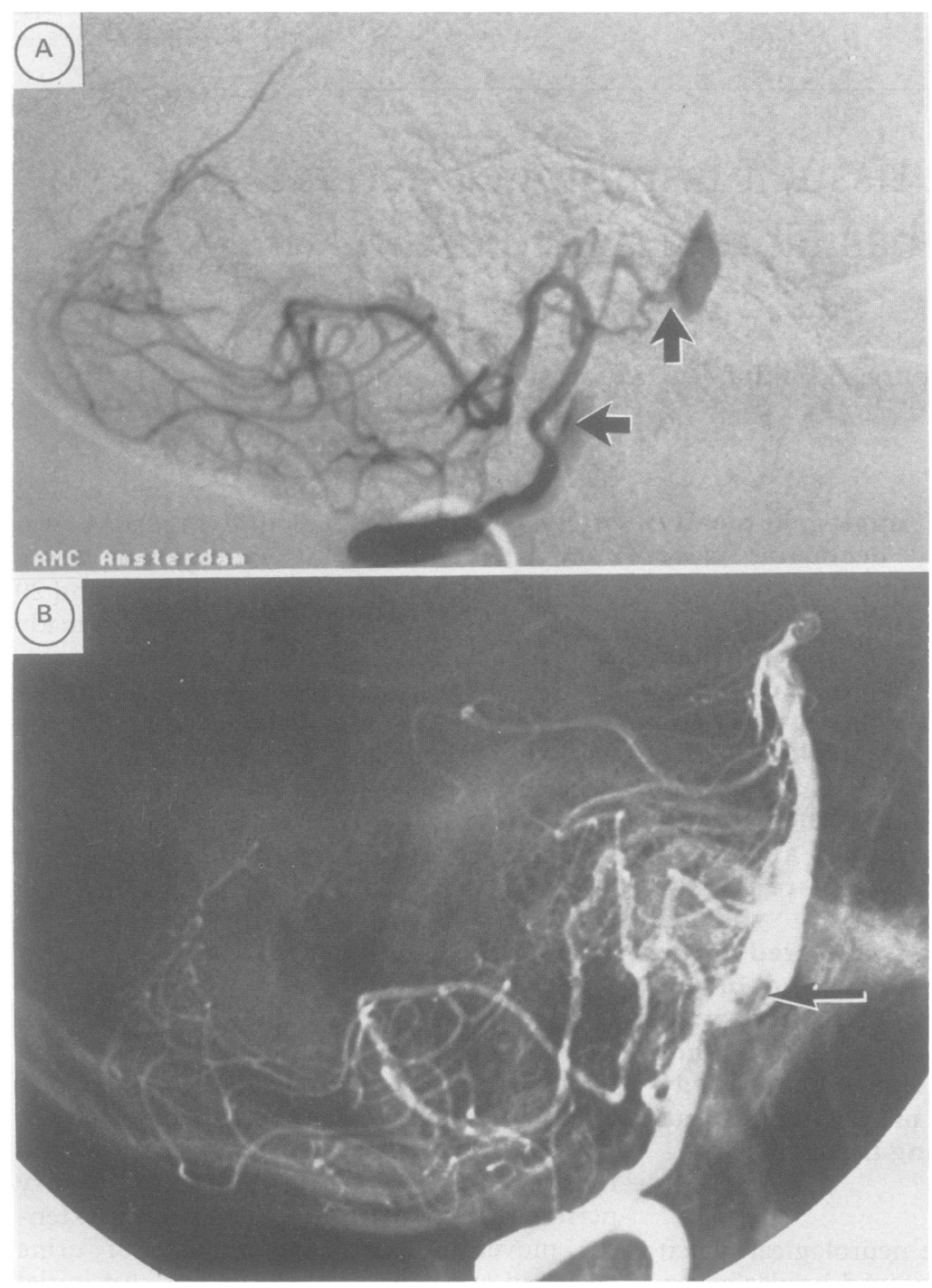

Figure 1a Left vertebral intraarterial subtraction angiography, lateral view, before treatment of patient 2, showing caudal occlusion (arrow) of the basilar artery with some collateral circulation to the rostral part (arrow).

Figure $1 b$ Repeat angiography of patient 2, after six hours, documents reperfusion with remaining intraluminal thrombus (arrow) and poststenotic enlargement of the basilar artery.

Figure 2 ACT scan of patient 2, after two months, shows a small right occipital infarction. decerebrate extensor movements of the arms and finally apnoea.

The medical history showed hypertension and hypercholesterolaemia; the patient was a cigarette smoker. A CT scan showed a hyperdense basilar artery and an old supratentorial lacunar infarction. Angiography disclosed a congenitally absent right vertebral artery and, after selective catheterisation of the left vertebral artery, a proximal occlusion of the basilar artery with some collateral circulation to the distal part (fig 1a). Treatment with rtPA was started immediately, 130 minutes after the onset of stroke, with a remarkable and sudden improvement in half an hour. Three hours after initiation of therapy neurological examination was normal, except for a left upper quadrantanopia, which gradually diminished in the next few weeks. Repeat angiography after six hours showed recanalisation with a local stenosis (fig 1b). Eight weeks later a small right occipital infarction was visible on CT (fig 2).

\section{Discussion}

Our first patient was treated at six hours after onset of symptoms. There was no recanalisation on repeat angiography, and his condition deteriorated and he died. At necropsy there was a pontine haemorrhagic infarction with haematoma formation. The second patient was treated after 130 minutes and had a successful recovery. In our opinion early repeat angiographic examination is necessary in the pilot phase of rtPA treatment in this condition, as the quintessence of thrombolytic therapy is the question of whether reopening of occluded vessels is accompanied by clinical improvement. With large delays of repeat angiography spontaneous recanalisation may have occurred, and a causal relationship with treatment is less certain. However, even recanalisation after six hours can be the result of a natural course instead of treatment.

Our finding of no improvement in the patient in whom recanalisation was not achieved, and recovery during rtPA treatment in the patient with recanalisation suggests a relationship between, a very likely rtPAinduced, reperfusion and clinical recovery. Haemostatic parameters, plasminogen and alpha-2-antiplasmin, before treatment and reassessed after 12 hours, showed evidence of consumption of clotting factors, comparable in both patients. Time span of the occlusion, with organisation of the clot, might be important, but on the other hand characteristics of the original clot itself can be important, with a "white", thrombocyte rich clot less amenable to dissolution than a "red", fibrin rich clot. ${ }^{7}$ Angiographically proved reopening of coronary vessels does not occur in $30-40 \%$ of patients treated with systemic rtPA. ${ }^{6}$ Pathological examination of the first patient showed localised intimal thickening of the basilar artery with an already partially organised thrombus.

Complications in the first patient included secondary haemorrhagic transformation of a 
large pontine infarction and bleeding from puncture sites. No other complications in this patient who had severe vascular disease were encountered at necropsy. After a period of six hours of total occlusion there may be too much damage to vessel walls and systemic rtPA may only augment the risk of local bleeding, as in the first patient. The second patient may have experienced a complication which has not previously been reported in thrombolysis of cerebral thromboembolism: an embolus was dislodged from the occluded basilar artery. The embolus was apparently not itself dissolved by rtPA and may have caused the occipital infarction. Spontaneous artery-to-artery embolism from vertebrobasilar atherosclerotic lesions causing occipital stroke has been described before. ${ }^{89}$ On repeat angiography in the second patient an intraluminal clot of the basilar artery was visible; risk factors for atherosclerotic arterial disease were found and treated. ${ }^{10}$ Oral anticoagulation was continued. Involuntary movement at onset caused a long diagnostic delay in the first patient. These movements occurred in the second patient too, but were recognised adequately. These convulsive-like movements are probably a sign of acute "vascular decerebration". ${ }^{11}$
The use of rtPA is a promising development in basilar thrombolysis, but has limitations in effectiveness in dissolving all clots, and may have a causative or additive part in haemorrhagic transformation of large infarction, when administered after too much delay.

1 Patterson JR, Grabois $M$. Locked-in syndrome: a review of 139 cases. Stroke 1986;17:758-64.

2 Archer CR, Horenstein S. Basilar artery occlusion. Clinical and radiological correlation. Stroke 1977;8:383-90.

3 Hacke W, Zeumer H, Ferbert A, Bruckmann H, del Zoppo GJ. Intra-arterial thrombolytic therapy improves outcome in patients with acute vertebrobasilar occlusive disease. Stroke 1988;19:1216-22.

4 Henze T, Boeer A, Tebbe U, Romatowski J. Lysis of basilar artery occlusion with tissue plasminogen activator. Lancet 1987;1:1391.

5 Neuhaus KL, Feuerer W, Jeep-Tebbe S, Niederer W, Vogt A, Tebbe U. Improved thrombolysis with a modified dose A, Tebbe U. Improved thrombolysis with a modified dose regimen of recombinant tissue-type plas
activator. $J$ Am Coll Cardiol 1989;14:1566-9.

6 Marder VJ, Sherry S. Thrombolytic therapy: current status (first of two parts). N Engl J Med 1988;318:1512-20.

7 Jang IK, Gold HK, Ziskind AA, et al. Differential sensitivity of erythrocyte-rich and platelet-rich arterial thrombi to lysis with recombinant tissue-type plasminogen activator. Circulation 1989;79:920-8.

8 Koroshetz WJ, Ropper AH. Artery-to-artery embolism causing stroke in the posterior circulation. Neurology 1987;37:292-6.

9 Pessin MS, Lathi ES, Cohen MB, Kwan ES, Hedges TR, Caplan LR. Clinical features and mechanism of occipital infarction. Ann Neurol 1987;21:290-9.

10 Levine SR, Ouint DJ, Pessin MS, Boulos RS, Welch KMA. Intraluminal clot in the vertebrobasilar circulation: Clinical and radiological features. Neurology 1989;39:515-22.

11 Ropper AH. "Convulsions" in basilar artery occlusion. Neurology 1988;38:1500-1. 